\title{
Creating Smart and Adaptive Medical Devices: Device Data and Analytics
}

Bruce Reiner I*

Department of Radiology, Veterans Affairs Maryland Healthcare System, 10 North Greene Street, Baltimore, Maryland, USA

*Corresponding author: Bruce Reiner I, Department of Radiology, Veterans Affairs Maryland Healthcare System, 10 North Greene Street, 11402 Newport Bay Drive, Berlin, Maryland 21811, USA, Tel: 4435134201; E-mail: breiner1@comcast.net

Received date: June 25, 2017; Accepted date: July 20, 2017; Published date: July 30, 2017

Copyright: (c) 2017 Reiner $\mathrm{BI}$, et al. This is an open-access article distributed under the terms of the Creative Commons Attribution License, which permits unrestricted use, distribution and reproduction in any medium, provided the original author and source are credited.

Keywords: Biosensors; Medical devices; Diagnosis; Medicine; Thrombosis; Microbiology

\section{Introduction}

In the current state of medical device technology and implementation, most implanted medical devices are relatively static and utilitarian in design and function, with single use functionality and data. As a result, implanted medical devices tend to be relatively unresponsive to their local environment, which often leads to device related complications which can result in device removal, patient morbidity, and requirement for systemic therapy (with its own association risks and complications).

In an earlier related article [1], a proposed innovation was described in which medical device embedded miniaturized biosensors could create an opportunity to produce "smart", "intelligent", and "adaptive" medical devices capable of continuously collecting objective and standardized data related to device functionality, structural integrity, and the local environment; with the goal of facilitating early diagnosis and treatment of device related complications.

With the ever growing diversity and functionality of biosensors, the data possibilities and derived analytics could become quite expansive and potentially improve medical device performance, lifetime, and overall safety. At the same time, the ability to prospectively record standardized device related data could result in the creation of large multi-institutional referenceable device databases which could assist in the creation of device, patient, and context specific best practice guidelines; which can further promote the existing goals of evidence based and personalized medicine.

The same opportunities offered through real-time analysis of sensor embedded medical devices can be extended to include synchronous data of other medical devices, along with complementary clinical data available in the patient electronic medical record. The end goal is to produce a multi-source data network which can communicate via wireless technology to facilitate real-time data analytics, patient and provider communication, computerized decision support, and customizable intervention.

\section{Data Components and Analyses}

While the variety and number of biosensor derived data elements are rapidly expanding with continuous innovation in biosensor technologies, the pertinent medical device data can be grouped into 3 broad categories: functional, structural, and environmental data. Functional data is specific to the individual medical device and its intended purpose (e.g. vascular patency, anatomic fixation) and essentially measures the effectiveness of the device in maintaining homeostasis. Structural data refers to the mechanical stability and integrity of the device and its individual components. Environmental data refers to the local cellular and tissue environment in which the implanted device resides and is a frequent source of device related complications. These complications can fall into two categories; generalized and device specific. Generalized complications are in large part a concern for most different medical devices and include infection, cellular proliferation, and bleeding. Device specific complications are in large part unique to the individual type of device and its anatomic location. For discussion purposes, this manuscript will focus on generalized complications since they are more applicable to the broad population and diversity of medical devices in general.

The representative functional and structural data elements provided are specific to the device clinical performance and structural integrity. The principle types of local environmental complications (i.e., infection, thrombus, cellular proliferation) listed would in turn each have associated data elements (e.g. cytokines for infection, fibrinogen for thrombosis, endothelial and smooth muscle cell-derived neuropilin-like protein for cell proliferation). A key feature in the data analysis is the ability to continuously record prospective data over the lifetime of the medical device; thereby providing for early detection of data variation through temporal analysis. As data is collected over large patient cohorts, the expected range of device-specific data variability can be defined; providing greater specificity to the analysis.

The detection of a data outlier can also result in modification to the routine data collection process by increasing the frequency of device data collection, increasing the vigilance of sensor quality control, and increasing correlation with pertinent electronic medical record (EMR) data. In the example of suspected device infection, relevant data from the EMR may include vital parameters (e.g. temperature), laboratory data (e.g. white blood cell (WBC) count and differential), imaging exams (e.g. nuclear medicine WBC scan), and microbiology (e.g. blood culture). Rules based analysis and computerized decision support tools can be created specific to individual data elements to assist in analysis and automate the data extraction process.

Knowledge of sensor distribution within the individual medical device would also play a key role in data collection and analysis. When abnormal data is collected within a limited number of device sensors, the device-specific sensor roadmap would provide specific location of the sensors providing the abnormal data of record. The ability to accurately localize abnormal data takes on heightened importance in early detection and intervention. In the example of an arterial stent, localized sensor-derived data abnormalities could identify early signs of infection, thrombus, or a structural defect; all of which would benefit from immediate and localized intervention in the hopes of maintaining device function and avoiding significant patient morbidity. In current practice such early and localized diagnosis and intervention is routinely not practical and often results in systemic therapy and/or device removal. As described in a companion article 
[2], sensors provide both diagnostic and therapeutic options, and have the ability to store and inject drugs (e.g. antimicrobials, thrombolytics) locally, with the potential to reduce the negative clinical effects of systemic therapy.

\section{Multi-Device Data}

Given the diversity and multi-functionality of medical devices, multiple synchronous medical devices are commonly encountered; particularly in the acutely ill, elderly, and chronic disease patient population [3-5]. In the circumstances where multiple devices of similar functionality or organ system are simultaneously present in different anatomic locations, the device from each individual device is complementary to its counterpart devices; creating the opportunity for synergistic multi-device data analysis.

Using the cardiovascular system as an example, an individual patient with arterial occlusive disease may have multiple implanted arterial stents (of different design and structure) coexisting in the coronary arteries, aorta, visceral, and lower extremity arteries. The ability to correlate synchronous data from these multiple anatomic locations may provide insights which cannot be achieved through single device data analysis alone. To illustrate how multi-device data may prove complementary and facilitate early diagnosis we will take the example of arterial stents simultaneously located in the abdominal aorta, right common iliac artery, and bilateral superficial femoral arteries (Insert Diagram). Biosensor derived flow related data (e.g. pressure, velocity, turbulence) in the right common iliac arterial stent demonstrates consistent measurements throughout the stent length, indicating stent patency and proper performance. However, when the arterial inflow data (i.e., sensors in the proximal stent) is correlated with data from the abdominal aortic stent (which is proximal to the common femoral artery stent), then one can detect there is a drop off in arterial pressure somewhere between the distal end of the abdominal aortic stent graft and the proximal end of the right common femoral arterial stent. The severity of this obstruction can be further surmised based upon the degree of segmental pressure change between these two arterial stents.

If we were to go one step further and compare the pressure and velocity measurements between the right and left common femoral arterial stents, we find that the inflow measurements of the left common femoral artery stent are comparable to the pressure/velocity outflow measurements of the abdominal aortic stent graft. These comparative device specific measures provide evidence that the obstruction occurs after (i.e., distal to) the aortic bifurcation and proximal to the right common femoral arterial stent, most likely at the origin of the right common femoral artery. If the obstruction had instead been located in the distal abdominal aorta (proximal to the aortic bifurcation), a comparable abnormality would have been expected in the left common femoral artery stent, which was not the case. At the same time, comparative pressure and flow inflow measurements in the right superficial femoral artery stent show no significant change in measurements when compared to the right common femoral artery stent, which would mitigate against an obstruction in the arterial segment separating these two stents.

Now let us go one step further in order to identify a critical application of the invention by showing how the device related measurements can be sequentially analyzed to identify the timing, severity, location, and etiology of pathology. Using the same patient with 4 arterial stents (in the treatment of peripheral vascular disease) we now have an example of a sudden and rapid change in arterial velocity inflow measurements in the right common femoral artery stent, accompanied by complete absence of distal stent outflow. This indicates that an acute obstruction has occurred in the right common femoral artery stent, the specific location of which can be determined by analyzing neighboring sensor data along the course of the stent. The two most likely causes of pathology are progression in atherosclerotic plaque or embolism. Since the "pre-event" measures showed a relatively mild degree of obstruction and the abnormity occurred quite acutely (i.e., in the 15 minute interval of routine sequential measurements), the logical etiology is that of embolism. Since the embolism source can occur anywhere proximal to the point of obstruction it is often difficult to localize. However in this case, analysis of the sensors in the internal wall of the abdominal aortic stent graft had previously demonstrated a significant burden of atherosclerotic plaque along the middle of the stent which is no longer detected. By measuring the distance between adjacent sensors in the abdominal aortic stent graft "before and after" sensor data, one can estimate the size of the embolus (i.e., $2.5 \mathrm{~cm}$ ), which correlates with the luminal diameter of the occluded right common femoral artery stent. Knowing the etiology, source, timing, and severity of this obstruction can provide timely diagnosis, notification, and intervention. Having the ability to correlate real-time data from multiple individual devices provides additional knowledge and insight not available when data is limited to that of a single medical device alone.

Using another example, suppose this same patient had an indwelling cardiac pacemaker due to an underlying cardiac arrhythmia. Analysis derived from the pacemaker sensors revealed a prolonged period of atrial fibrillation 24 hours prior to the event in question (i.e., embolic obstruction of the right common femoral artery stent). Since atrial fibrillation is a well-documented cause for cardiac thrombus formation and subsequent emboli, this could also serve as a source of the embolic disease. One method of differentiating between the two possible sources of emboli (i.e., cardiac versus abdominal aorta) is to review the flow data derived from the abdominal aortic stent graft data during the specific time frame of concern (i.e., the period of time immediately preceding and up to the time the occlusion of the right common femoral artery stent was identified). If, the thrombus had originated from the heart, then the embolus would have had to pass through the abdominal aortic stent before passing into and obstructing the right common arterial stent graft. This could have been identified by retrieving sensor derived data within the abdominal aortic stent graft during the time in question and evaluating for the presence of abnormal internal flow (e.g. loss of normal laminar flow, alteration in flow directionality, presence of a new intraluminal mass). This last feature can be facilitated by incorporating ultrasound capabilities within the sensors, which provides the ability to use ultrasound to analyze medical device internal flow and wall characteristics.

While these examples illustrate the potential for analysis of analogous multi-device data, similar opportunities exist for correlating data from disparate devices, whose functionality and organ system differ from one another. For illustrative purposes we will take the example of an abdominal aortic stent graft and an artificial pancreas. The aortic stent graft serves to maintain arterial flow and integrity of the abdominal aorta, while the artificial pancreas serves to monitor blood glucose and dynamically excrete insulin in an attempt to maintain endocrinology homeostasis. In this example, biosensors in the aortic stent graft detect small increases in infection-related data measurements (e.g. cytokines, white blood cell aggregation), which cause concern for a developing device-related infection. This prompts 
an automated query for retrieval of infection related laboratory data from the EMR, along with an automated alert for infection related data in other medical devices. Upon recognition of this alert, the data associated with the artificial pancreas is reviewed to detect any temporal changes in data which may be directly or indirectly related to early infection. This data analysis demonstrates that recent blood glucose levels have unexpectedly increased beyond the patient normal range and required progressively increased levels of insulin over the past 24-36 hours. Combining these two disparate device data sets yields an association; glucose levels often acutely increase in the setting of infection. Other causes of unexpected acute rise in glucose levels include medication change, stress, non-functioning insulin or insulin pump, and dietary change. An automated query of the EMR and artificial pancreas database effectively excludes the other possible etiologies and confirms infection as the most likely etiology. With localized release of antibiotics (at the specific sensor location of concern), both the infection-related data measures and glucose/insulin levels return to normal. This example illustrates how data from disparate medical devices can be collectively analyzed and acted upon to improve diagnosis and treatment.

\section{Conclusion}

The creation of standardized and objective medical device data (through embedded miniaturized biosensors) provides the ability to create referenceable medical device databases, which can comingle multi-institutional data for large sample size statistical analysis. When taking into account specific patient attributes and the clinical context, retrospective analysis of the database can assist in selection of the medical device and clinical institutional providers. The creation of realtime sensor data within the medical device provides the ability to prospectively analyze device functionality, structural integrity, and the local environmental complications. When this device-specific real time data is correlated with historical device and clinical outcomes data; predictive analytics can be performed which provide insight as to the relative risk of device malfunction and/or clinical complications. When the medical device data is combined with artificial intelligence and automated data extraction from the electronic patient record, computerized decision support tools can be created in an effort to improve diagnosis and treatment of device related complications.
In addition to analysis of an individual medical device, data from both analogous and disparate medical devices can be collectively correlated and analyzed to enhance diagnosis and treatment decision making. This multi-device data analysis has the potential to identify new and unexpected interaction effects between devices and the relative impact environmental influences have on different device types and anatomic locations. By embedding biosensors within medical devices, one can transform the device from a passive and static instrument to one of dynamic and adaptive features, which create and analyze device related data in real-time, thereby offering the potential to improve device performance and healthcare outcomes.

\section{Acknowledgments}

We greatly appreciate Addis Ababa University for supporting this study. We are also grateful to the Department of Medical Laboratory Sciences that gave us ethical clearance for this study. Our deep gratitude goes to those study participants and their parents/guardians who showed their willingness to participate in this study by giving written consent.

\section{Conflict of Interest}

The authors declare that they have no competing interests.

\section{References}

1. Reiner B. Creating smart and adaptive medical devices. Part 1: embedding miniaturized biosensors.

2. Reiner B. Creating smart and adaptive medical devices. Part 2: the biofilm-medical device conundrum.

3. Mylotte D, Osnabrugge RLJ, Windecker S, Lefèvre T, de Jaegere $P$, et al. (2013) Transcatheter aortic valve replacement in Europe: adoption trends and factors influencing device utilization. J Am Coll Cardiol 62: 210-219.

4. Polderman KH, Girbes ARJ (2002) Central venous catheter use. Part 2: infectious complications. Intensive Care med 28: 18-28.

5. Nowygrod R, Egorova N, Greco G, Anderson P, Gelijns A, et al. (2006) Trends, complications, and mortality in peripheral vascular surgery. J Vasc Surg 43: 205-216. 